\title{
Flexible protective DLC films on rubber: fundamental concepts and applications
}

\author{
Y. T. Pei, X. L. Bui, D. Martinez-Martinez, J. P. van der Pal \\ \& J. Th. M. De Hosson \\ Department of Applied Physics, Materials Innovation Institute M2i, \\ University of Groningen, The Netherlands
}

\begin{abstract}
Dynamic rubber seals are major sources of friction of lubrication systems and bearings, which may take up to $70 \%$ of the total friction. The solution we present is to coat rubber with DLC thin films by which the coefficient of friction is reduced from above 1.5 to below 0.15 . Coating rubber is very challenging because the film/coating must be flexible and strongly adhered to the surface. Here we present and discuss our novel approach by depositing flexible DLC films on various rubbers via self-segmentation. By making use of the substantial thermal mismatch between DLC film and rubber substrates a dense crack network forms in DLC films and contributes to flexibility. The size of film micro-segments can be tuned by varying the bias voltage of pulsed-DC plasma CVD, which governs the amplitude of the substrate temperature variation during deposition. An analytical model is developed to predict the crack spacing (equivalent to the size of film segments) and the result fits well to the measured data. The formation mechanism of crack network and its effect on the flexibility and friction of DLC film coated rubbers are scrutinized. This paper provides generic design rules for the deposition of flexible and ultra-low friction films on rubber seals and the approach can drastically reduce the energy consumption in bearings and lubrication systems.
\end{abstract}

Keywords: DLC film, flexibility, rubber substrate, magnetron sputtering, tribology. 


\section{Introduction}

For a rigid protective film deposited on compliant substrates such as rubbers, the most critical issues that determine the performance of the entire system are the film flexibility and interfacial adhesion. Sufficient flexibility of the protective film is required to adapt large deformation of rubber substrates under loading and can be achieved through film segmentation. Strong adhesion is a necessary condition ensuring that the segments of the film adhere well on the rubber substrates maintaining functionality under loading condition. In particular the combination of flexibility and strong adhesion is crucial for DLC films coated on dynamic rubber seals where cyclic and large elastic deformation may be exerted.

In the past a tile-like structure for films on rubber was proposed and deposited by using a net mask in front of the substrate [1]. Obviously, there are technical problems with this approach: the size of film segments is rather limited to submillimeter and especially the open gap of size at least tens of micrometers (equal to the thickness of the grid). Experimental results revealed that the impact between the asperity on the surface of sliding counterpart and the sharp edges of the open cracks in the films produced a large amount of debris that lead to a high friction and severe wear of coated rubber [2]. In this paper, we report another approach for depositing micro-segmented DLC films of superior flexibility on rubber. The novelty is that the size of film segments separated by close crack network can be well controlled at much smaller length scales. The formation mechanism of crack network during deposition and its effect on the friction of DLC film coated rubber are presented and reviewed [3].

\section{Experimental}

Hydrogenated nitrile butadiene rubber (HNBR) sheet of $2 \mathrm{~mm}$ thickness was used as substrate. The brown HNBR substrates of $45 \times 45 \mathrm{~mm}^{2}$ size were first cleaned by detergent in an ultrasonic cleaner and then rinsed with demineralized water. Thereafter, wax removal was carried out in an ultrasonic tank with hot demineralized water $\left(90-95^{\circ} \mathrm{C}\right)[4]$. This washing process with hot water was repeated three times. Next, the HNBR substrates were dried in a centrifugal machine and then heated up to $120{ }^{\circ} \mathrm{C}$ for 15 minutes in order to evaporate all absorbed water. The substrates were cooled down in ambient air to room temperature before being loaded into the deposition chamber. The instrumental modulus of HNBR rubber, as determined by the method described elsewhere [5], is $10.2 \mathrm{MPa}$ and its surface roughness is $0.35 \mu \mathrm{m}$ measured after wax removal using laser confocal microscopy. The HNBR has a typical coefficient of thermal expansion $180 \times 10^{-6} \mathrm{~K}^{-1}$.

Plasma cleaning treatment of rubber substrates and deposition of DLC films were carried out in a Teer UDP400/4 closed-field unbalanced magnetron sputtering system, which was configured of four magnetrons that were all powered off. HNBR substrates were cleaned by Ar plasma for 30, 35, 40 and 45 minutes at pulsed-DC $(250 \mathrm{kHz}, 87.5 \%$ duty cycle) bias voltage of $-600 \mathrm{~V}$, $-500 \mathrm{~V},-400 \mathrm{~V}$ and $-300 \mathrm{~V}$, respectively. During the last ten minutes of plasma 
cleaning, hydrogen gas was added (50\%) for reactive plasma cleaning to enhance the interface adhesion of DLC films. Immediately after the plasma cleaning treatment, DLC films were deposited by plasma CVD (p-CVD) at pulsed-DC bias voltage of $-300 \mathrm{~V}$ and $-400 \mathrm{~V}$, respectively, for 120 minute and 60 minute to reach the same film thickness of $300 \mathrm{~nm}$. The ratio of gas flow rates was set at $\operatorname{Ar}: \mathrm{C}_{2} \mathrm{H}_{2}=3: 2$, and at constant process pressure of $3 \times 10^{-3}$ mbar. However, the residual hydrogen in the inlet pipeline used also for acetylene might last till the first 10 minutes of deposition, during which period the growth of DLC film was only minor. The HNBR substrates were rotated at a revolving speed of $3 \mathrm{rpm}$ during the deposition. The substrate temperature during plasma etching/ deposition was measured in situ with a thermal couple inserted into the rubber sheet substrate.

The flexibility and adhesion strength of the DLC film were examined via insitu stretch tests on coated specimens of gauge section dimensions $10 \times 3 \times 2 \mathrm{~mm}^{3}$, with a tensile stage installed in a Philips XL-30 FEG scanning electron microscope (SEM). The coated rubber sheets were glued onto $\varnothing 30 \mathrm{~mm}$ polished M2 steel discs for tribotests. The tests were performed at room temperature (20$23{ }^{\circ} \mathrm{C}$ ) on a CSM high temperature tribometer with ball-on-disc configuration. The counterpart was a commercial $ø 6 \mathrm{~mm} 100 \mathrm{Cr} 6$ steel ball of hardness HRC 60-62. All the tribotests were carried out at a sliding velocity of $10 \mathrm{~cm} / \mathrm{s}$ and a constant humidity of $35 \pm 1 \%$ kept with a humidity regulator. The surface morphology and wear track of DLC film coated rubber after stretch and tribotests were characterized with SEM.

\section{Results and discussion}

The measured temperature evolution of rubber substrates during plasma cleaning and successive deposition is shown in Fig. 1a. The temperature variation $(\Delta T)$ exposed on HNBR substrates during deposition is related to the substrate temperature at the beginning of deposition and the bias voltage used for the pCVD process. Assuming that the plasma can be considered as a 'hot body' whose temperature ( $\left.T_{\text {plasma }}\right)$ is constant and merely dependent on the bias voltage applied. The heat flow from the "hot" plasma to the "cold" substrate, which changes the temperature of substrate as a result, depends on the temperature gradient between the plasma and the substrate. From Fourier's heat conduction equation it follows that

$$
T_{\text {substrate }}=\left(T_{0}-T_{\text {plasma }}\right) \times e^{-K t}+T_{\text {plasma }}
$$

where $T_{0}$ is the initial temperature of rubber substrate. The measured substrate temperatures versus time of plasma treatment (cleaning and deposition) can be well fitted by Eq. (1), as indicated by the solid curves in Fig. 1(a). By finding the exponential factor $\mathrm{K}$ for different rubbers, various amplitudes of $\Delta T$ (the temperature variation during the whole process of film deposition) can be readily designed for the control of segmentation (as to be discussed in the following) by changing the bias voltage against deposition time. 

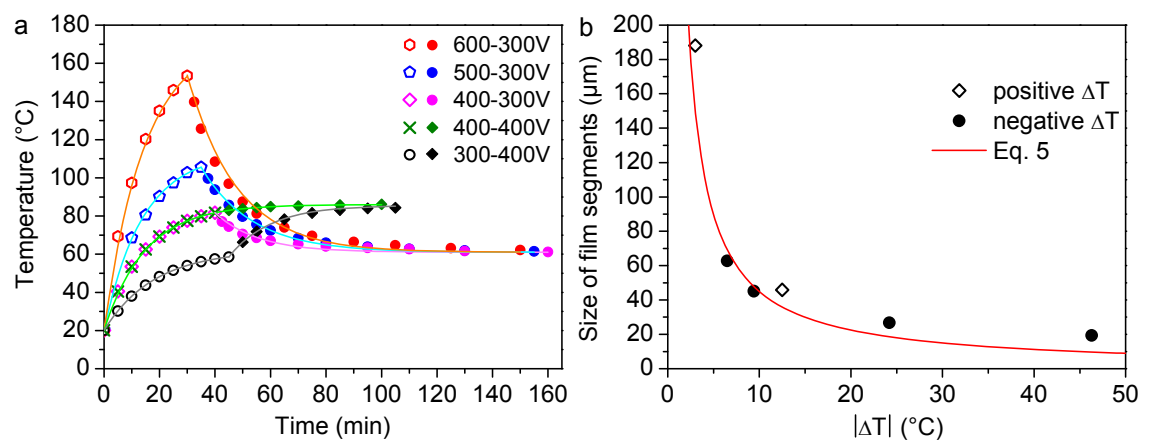

Figure 1: (a) Evolution of measured substrate temperature versus time of plasma cleaning (open symbols) and deposition (close symbols), with the solid curves fitted according to Eq. (1); (b) Size of film segments measured and predicted with Eq. (5) versus temperature variation $\Delta T$ exposed during deposition.

The surface morphology of DLC thin films on HNBR deposited under the condition of varying substrate temperature is characterized by crack networks, as shown in Fig. 2. The effective temperature variation exposed during deposition governs the expansion or shrinkage of rubber substrates and thus the mismatch strain in a growing DLC film, which determines in turn the density of the crack network or the size of DLC film segments. It is clear that a positive $\Delta T$ or tensile stress in the growing DLC film leads to the formation of a crack network and that the size of film segments is related to the amplitude of $\Delta T$ (Figs. 2a and 1b). In contrast, a negative $\Delta T$ or compressive stress results in the formation of wrinkles but also leads to a crack network whose density is likewise governed by the amplitude of $\Delta T$ (Figs. $2 \mathrm{~b}$ and $1 \mathrm{~b}$ ). The different states of deformation are reflected in the morphology of segments: the film segments formed under tensile misfit stress (positive $\Delta T$ ) are rather flat (see Fig. 2a), and those generated under compressive stress (negative $\Delta T$ ) are curved (convex) as shown in Fig. 2b. It is anticipated that the cracks originate in the early stage of DLC film growth due to the thermal mismatch strain once a critical thickness is reached [6,7], and continuously grow with increasing the film thickness. The result is very interesting since the segment size of DLC films is on a micrometer scale and can be tuned in a controllable manner. In this way we are able to control the density of crack network or wrinkles, without the necessity of using a net mask as proposed in earlier work [1]. Especially, the cracks are close and their edges bend inwards (see Fig. 2), which are crucial for preventing the formation of a large amount of wear debris that causes wear and high friction in practical applications [2].

The size of the film segments are related to the thermal mismatch strain that depends on the $\Delta T$ exposed on rubber substrate during deposition. The thermal mismatch strain between a DLC film and rubber substrate arises due to the 

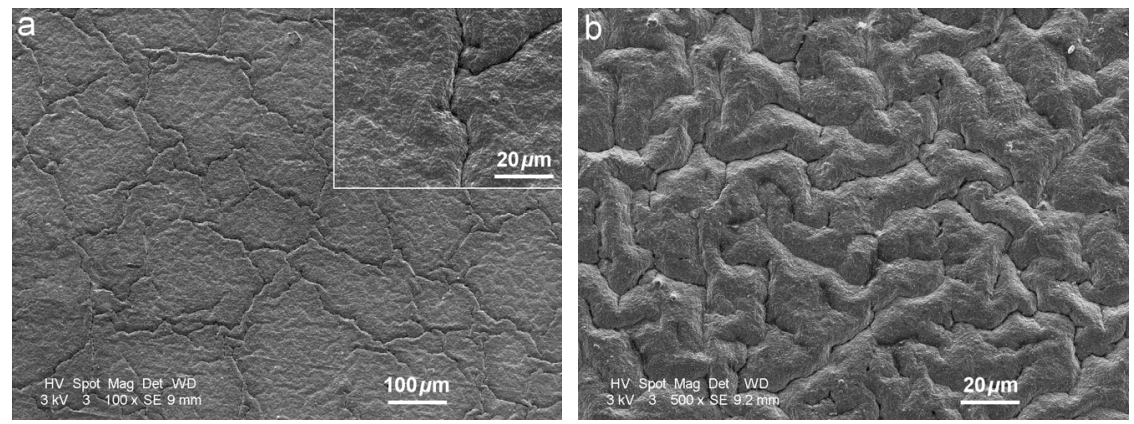

Figure 2: $\quad$ Surface morphology of DLC films on HNBR deposited at different temperature variations: (a) $\Delta \mathrm{T}=3.0{ }^{\circ} \mathrm{C}$ with an inset of higher magnification showing the inward bended crack edges; (b) $\Delta \mathrm{T}=-46.3{ }^{\circ} \mathrm{C}$.

considerable difference in their coefficient of thermal expansion (CTE, $\alpha)$ and is usually described as:

$$
\Delta \varepsilon=\left(\alpha_{s}-\alpha_{f}\right) \Delta T
$$

where the subscripts $f$ and $s$ indicate the film and substrate, respectively. The crack spacing of a film having identical elastic properties as the substrate can be estimated as [7]:

$$
l \cong \frac{5.6}{\varepsilon_{0}} \sqrt{\frac{\Gamma_{f} t_{f}}{\bar{E}}}
$$

where $\Gamma_{f}$ and $t_{f}$ are the fracture energy and thickness of the film, and $\varepsilon_{0}$ the applied strain or mismatch strain. For DLC film coated rubber where the two materials have rather different elastic properties, the effective modulus $\bar{E}$ for equal biaxial stress condition needs to be modified so as to take into account the differences in properties and the thickness ratio between the DLC film and the rubber substrate [8]:

$$
\bar{E}=\frac{E_{s} t_{s}}{E_{s} t_{s}+E_{f} t_{f}} \frac{E_{f}}{1-v_{f}}
$$

where $E$ is the modulus, $v$ Poisson's ratio and $t$ the thickness of DLC film or rubber substrate.

Inserting Eqs. (2) and (4) into Eq. (3) yields

$$
l \cong \frac{5.6}{\left(\alpha_{s}-\alpha_{f}\right) \Delta T} \sqrt{\frac{\Gamma_{f} t_{f}\left(1-v_{f}\right)\left(E_{s} t_{s}+E_{f} t_{f}\right)}{E_{f} E_{s} t_{s}}}
$$

Consequently, the crack spacing is inversely proportional to $\Delta T$, and also related to the fracture energy, effective modulus and thickness of DLC films. To verify the validity of Eq. (5), $\Gamma_{f}=35 \mathrm{~J} \mathrm{~m}^{-2}$ for DLC films [9] is used (other physical constants are listed in Table 1). We have estimated the average crack 
spacing (equal to the average size of film segments), and good agreement between experiment and theory is attained (see Fig. 1(b)).

Table 1: $\quad$ Modulus $(E)$, thickness $(t), \operatorname{CTE}(\alpha)[10]$ and Poisson's ratio $(v)$ of HNBR substrate and DLC film.

\begin{tabular}{ccccc}
\hline Properties & $E(\mathrm{MPa})$ & $t(\mu \mathrm{m})$ & $\alpha\left(\times 10^{-6} \mathrm{~K}^{-1}\right)$ & $v$ \\
\hline HNBR & 10.2 & 2000 & 180 & 0.5 \\
DLC film & 150000 & 0.3 & 6 & 0.13 \\
\hline
\end{tabular}

The density of crack network or segment size affects the flexibility and consequently the friction of DLC films coated HNBR rubber. Fig. 3b shows the tribological result of the DLC films of different segment sizes under the condition of dry sliding against $\varnothing 6 \mathrm{~mm} \mathrm{100Cr6}$ bearing steel ball: $1 \mathrm{~N}$ normal load, $10 \mathrm{~cm} / \mathrm{s}$ sliding speed, $35 \%$ relative humidity and room temperature. Clearly, the DLC films of smaller segments exhibit a lower steady-state coefficient of friction ( $\mathrm{CoF}$ ) and also shorter time to reach the steady-state CoF. In comparison, uncoated HNBR rubber exhibits a very high CoF about 1.3 1.5 under the same tribo-test conditions, see Fig. 3(a).

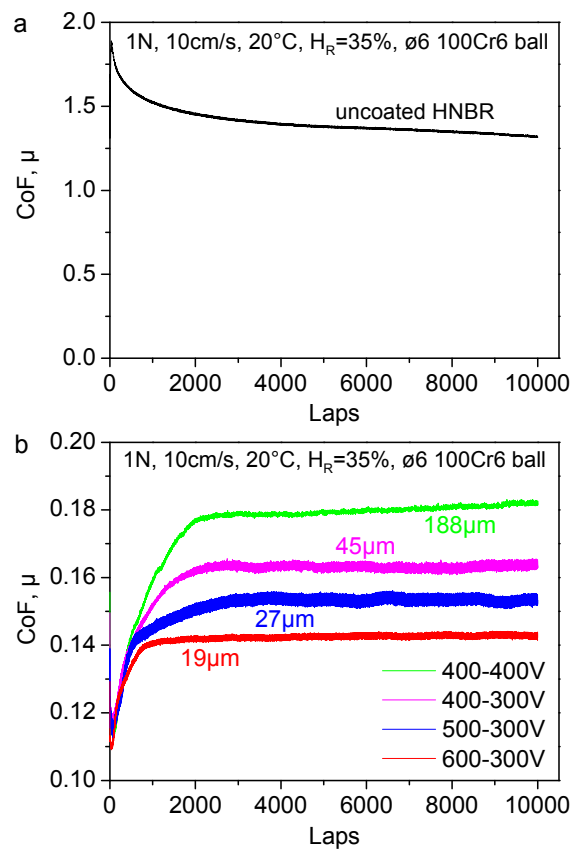

Figure 3: Coefficient of friction of uncoated HNBR (a) and $300 \mathrm{~nm}$ thick DLC films coated HNBR with segment sizes indicated (b).

SEM observations revealed that the DLC films with large segment sizes cracked further during sliding against steel ball, as shown in Fig. 4(a). These 
newly formed individual cracks propagate through the entire segments in a brittle manner and cause the formation of debris that wear both the counterpart and DLC film itself, leading to higher friction. In contrast, the DLC films with fine segment size (19 or $27 \mu \mathrm{m}$ ) hardly show new cracks under loading but only tiny polished spots on the convex segments after sliding for 10000 revolutions. More than $90 \%$ of the surface area of the DLC film was intact after a tribo-test. In-situ tension tests measured $8 \%$ strain tolerance for the DLC film of the smallest segment size, below which no new cracks were formed under tension. Rather, less than 5\% strain tolerance was observed for the DLC film of the largest segment size. Nevertheless, no delamination was observed in all the DLC films even after the rubber substrates broke down at $80 \sim 90 \%$ strain, indicating a superior interface adhesion. In addition to the experimental study, the result of machine tests confirms the superior performance of DLC film coated dynamic rubber seals in terms of ultra-low friction, long work life, energy saving and environment friendly solution.

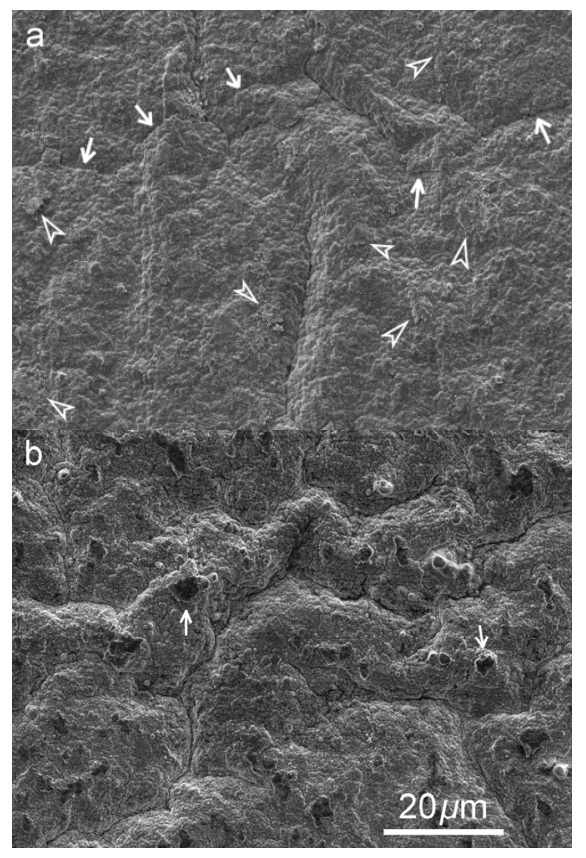

Figure 4: $\quad$ SEM micrographs showing the morphology of wear track of coated HNBR (1 N, $10 \mathrm{~cm} / \mathrm{s}, 10000$ lap): (a) DLC film of large segments (average size $188 \mu \mathrm{m}$ ), with the bold arrows indicating the long fracture cracks formed during tribo-test and the open arrows indicating the debris accumulated; (b) DLC film of small segments (average size $27 \mu \mathrm{m}$ ), with the arrows indicating tiny polished spots. 


\section{Conclusion}

Flexible DLC films of micrometer-scale segments have been deposited on rubber via self-segmentation. The size of film segments can be tuned by controlling the temperature variation of rubber substrate. The analytical description of crack spacing gives a good estimate of the segment size (equivalent to the crack spacing). Clearly the dense crack network contributes to the flexibility and ultralow friction of DLC coated rubber. The model and the experimental approach of film segmentation have been also applied to other kinds of rubber substrates, such as alkyl acrylate copolymer and nitrile butadiene rubbers, and the results confirm their validity. It can be concluded that this work provides generic design rules for the deposition of flexible and low friction films on dynamic rubber seals and an approach to drastically reduce energy consumption in bearings and other lubrication systems.

\section{Acknowledgements}

This research was carried out under the project number MC7.06247 in the framework of the Research Programme of the Materials innovation institute M2i (www.m2i.nl), Delft, the Netherlands. Financial support from the M2i is gratefully acknowledged. Dr. X.B. Zhou of the SKF Engineering \& Research Centre in Nieuwegein, The Netherlands is thanked for his valuable discussion and input.

\section{References}

[1] Aoki, Y. \& Ohtake, N., Tribological properties of segment-structured diamond-like carbon films. Tribology International, 37(11-12), pp. 941947, 2004.

[2] Pei, Y.T., Bui, X.L., Zhou, X.B. \& De Hosson, J.Th.M., Microstructure and tribological behavior of tungsten-containing diamondlike carbon coated rubbers. Journal of Vacuum Science \& Technology A, 26(4), pp. 10851092, 2008.

[3] Pei, Y.T., Bui, X.L. \& De Hosson, J.Th.M., Flexible protective diamondlike carbon film on rubber. Scripta Materialia, 63(6), pp. 649-652.

[4] Pei, Y.T., Bui, X.L., Mulder, E.D.G. \& De Hosson, J.Th.M., Adhesion improvement of hydrogenated diamond-like carbon thin films by predeposition plasma treatment of rubber substrate. Surface \& Coatings Technology, 203(14), pp. 1964-1970, 2009.

[5] Pei, Y.T., Bui, X.L., Zhou, X.B. \& De Hosson, J.Th.M., Tribological behavior of W-DLC coated rubber seals. Surface \& Coatings Technology, 202(9), pp. 1869-1875, 2008.

[6] Atkinson, A. \& Guppy, R.M., Mechanical stability of sol-gel films. Journal of Materials Science, 26(14), pp. 3869-3873, 1991.

[7] Thouless, M.D., Olsson, E. \& Gupta, A., Cracking of brittle films on elastic substrates. Acta Metallurgica et Materialia, 40(6), pp. 1287-1292, 1992. 
[8] Kadolkar, P.B., Watkins, T.R., De Hosson, J.Th.M., Kooi, B.J. \& Dahotre, N.B., State of residual stress in laser-deposited ceramic composite coatings on aluminum alloys. Acta Materialia, 55(4), 1203-1214, 2007.

[9] Wang, J.S., Sugimura, Y., Evans, A.G. \& Tredway, W.K., The mechanical performance of DLC films on steel substrates. Thin Solid Films, 325(1-2), 163-174, 1998.

[10] Marques, F.C., Lacerda, R.G. \& Champi, A., Thermal expansion coefficient of hydrogenated amorphous carbon. Applied Physics Letters, 83(15), pp. 3099-3101, 2003. 